\title{
Lokale Reflexivität und lokàle Dualität von Ultraprodukten: für halbgeordnete Banachräume
}

\author{
K.-D. KÜRSTEN
}

Es sei $L$ ein endlichdimensionaler Unterraum des dualen $\left(E_{i}\right)_{U}^{*}$ zum Ultraprọdukt geordneter Bánachräume. Dann existiert eine positive isometrische Einbettung von $L$ in $\left(E_{i}^{*}\right)_{V}$, die noch zusätzlichen Bedingungen genügt. Im- Falle von Banachverbänden kann die isometrische Einbettung als Verbandshomomorphismus gewählt werden. Für die nach dem Prinzip der lokalen Reflexivität existierenden fastisometrischen Einbèttungen endlichdimensionaler Uniterräume von $E^{* *}$ in $E$ ląsen sich entsprechende, jedoch etwas schwächere Positivitätseigenschaften erreichen.

Допустим что $\left(E_{i}\right)_{U}$ ултрапроизведение упоряддосениых банаховых пространс́тв и что $L$ конечномерное полиространство сопряженного пространства $\left(E_{i}\right)_{U}{ }^{*}$. Тогда "существует положительное изометрнческое нложение $L$ в $\left(E_{i}^{*}\right)_{U}$, которое удовлетворяет еще некоторым дополиительным условиям. В сілчае банаховых решеток изометрическое вложение можно взнть кақ гомоморфизм решеток. На существую́ие по принцпу локальной рефлексивности почти пзометрические вложения коненномерных подпространств $E^{* *}$ в $E$ можно накладывать соответственные, но несколько более слабые условия положительности.

Let $\left(E_{i}\right)_{U}$ be the ultraproduct of ordered Banach spaces and let $L$ be a finite dimensional subspace of the dual $\left(E_{i}\right)_{u}{ }^{*}$. Then there exists a positive isometric imbedding of $L$ into $\left(E_{i}^{*}\right)_{U}$ also satisfying some additional conditions, In the case of Banach lattices the isometric imbedding may be chosen as a lattice homomorphism. Similar but somewhat weaker positivity properties can be satisfied for the almost isometric imbeddings of finite dimensional subspaces of $E^{* *}$ into $E$ which exist by the principle of local reflexivity.

\section{Einleitun'}

Die in dieser Arbeit bewiesenen vier Theoreme haben mit dem Prinzip der lokalen Reflexivität von J. Lendenstraoss und H. P. Rosexthal [11] gemeinsam, daß die endliche Darstellbarkeit eines Banachraumes in einem bestimmten Unterraum behauptet wird, wobei noch zusätzliche Bedingungen erfültt sind. Beim Prinzip der. lokalen Reflexivität wird der Bidualraum $E^{* *}$ in $E$ endlich dargestellt, während die lokale Dualität von Ultraprodukten die endliche Darstellbarkeit des. Dualraumes eines Ultraproduktes im Ultraprodukt der Dualräume beinhältet. Theorem 1 ist in wesentlichen das bekannte Prinzip der lokalen Reflexivität, wobei zusätzlich eine Positivitätseigenschaft erreicht wird. Wie W. A. GEJLER und I. I. CuČEv zeigten, gilt dieses Theorem auch für lokalkonvexe Räume. Theorem 2 stammt im wesentlichen von J. L. Conroy und L.C. Moore jr. [2] und beinhaltet die lokale Reflexivität von .Banachverbänden. Die Theoreme 3 und 4 beinhalten die lokale Dualität von UItraprodukten von halbgeordneten Banachräumen bzw. von Banach verbänden. Die ersten Varianten von Theorem 3 wurden unabhängig in [10] und in [14] veröffentlicht. 'Theorem 4 wurde vom Autor zuerst auf der Konferenz zur Banachraumtheorie ' in Bukarest, September 1981, vorgestellt [9]. Für Varianten der Theoreme 1, 2 und 3 
sind eine Reihe anderer Beweise und Anwendungen bekannt (vgl. z. B. [1, 3, 6, 7]). Jie hier angegebenen Beweise beruhen alle auf einen Irennungssatz für eine Anzahl konvexer Mengen (Lemma 4). Die Arbeit wird durch einige Beispiele; die den Giiltigkeitsbereich der Theoreme abgrenzen, beendet. Bezüglich weiterer Eigenschaften von Ultraprodukten bzw. beziiglich elementarer Eigenschaften von Banachverbänden sei auf [6] bzw, auf [13] verwiesen.

\section{Bezeichnungén und Definitionen}

Es werden halbgeordnete Banachräume $E$ mit Positivitätskegel $K$ betrachtet. Im allgemeinen wird dabei nur verlangt, daß $K$ konvex und positiv homogen ist und die Null enthält. Ist $K \subset E^{\prime}$ bzw. $K^{\prime} \subset E^{*}$, so definieren wir die dualen Kegel

bzw.

$$
K^{+}:=\left\{f \in E^{*}: \dot{\operatorname{Re}} f(x) \geqq 0 \text { für jedes } x \in K\right\}
$$

$$
K_{+}^{\prime}=\left\{x \in E: \operatorname{Re} f(x) \geqq 0 \text { für jedes } f \in K^{\prime}\right\} \text {. }
$$

Fiir $C \subset E$ und $x \in E$ betrachten wir den Abstand

$$
d(x, C)=\inf \{\|x-y\|: y \in C\} .
$$

Ordnungsintervalle werden mit $[x, y]$ bezeichnet.

Mit $I$ bezeichnen wir eine Indexmenge und mit $U$ einen Ultrafilter auf $I$. Es sei cine Familie' $\left(E_{i}: i \in I\right)$ halbgeordneter Banachräume mit den Positivitätskegeln $K_{i} \subset E_{i}$ gegeben.

Zur Definition des Lltraprodukts (vgl. z. B. [6]) betrachtet man auf dem Raun $\left\{\left(x_{i}: i \in I\right): x_{i} \in E_{1}, \sup \left\|x_{i}\right\|<\infty\right\}$ die Halbnorm lim $\left\|x_{i}\right\|$. Der Faktorraum nach dem

Kern dieser Halbnorm wird Ultraprodukt der Räume $E_{i}$ bezüglich $U$ genannt und mit $\left(E_{i}\right)_{U}$ bezeichnet. Seine Elemente werden mit $\left(x_{i}\right)_{U}$ bezeichnet. Ein Element $\left(x_{i}\right)_{U}$ ist bereits definiert, wenn $x_{i}$ für alle $i$ aus einem Element des Ultrafilters definiert. und gleichmäßig beschränkt ist, denn man kann für die-restlichen $i$ setzen $x_{i}=0$. Eine bestinımite von $i \in I$ abhängige Alıssage heißt für fast alle $i$ erfüllt, wenn die Menge derjenigen $i \in I$, für die diese Aussage gilt, zum Ultrafilter gehört. Sind Teilmengen $C_{i} \subset E_{i}$ gegeben, so definieren wir

$$
\left(C_{i}\right)_{U}=\left\{\left(x_{i}\right)_{U}: x_{i} \in C_{i} \text { für fast alle } i\right\} \text {. }
$$

In $\left(E_{i}\right)_{U}$ wird der Positivitätskegel $\left(\dot{K}_{i}\right)_{U}$ betrachtet. Sind die Räume $E_{i}$ Banachverbände, so ist auch $\left(E_{i}^{\prime}\right)_{U}$ ein Banachverband, und es gilt $\left(x_{i}\right)_{U} \vee\left(y_{i}\right)_{U}=\left(x_{i} \vee y_{i}\right)_{U}$. Das Ultraprodukt der dualen Räume $\left(E_{i}^{*}\right)_{\dot{U}}$ wird durch die Formel

$$
\left(J\left(f_{i}\right)_{U}\right)\left(\left(x_{i}\right)_{U}\right)=\lim _{V} f_{i}\left(x_{i}\right)
$$

linear und isometrisch in $\left(E_{i}\right)_{U}{ }^{*}$ eingebettet. Dabei ist im allgemeinen $J\left(\left(E_{i}{ }^{*}\right)_{U}\right)$ $\neq\left(E_{i}\right)_{U}{ }^{*}$ und in den Theoremen 3 und 4 wird die Relation zwischen diesen beiden Räımen untersucht. Dabei werden noch folgende Bezeichnungen verwendet:

$$
\left(E_{i}\right)_{U}=G, \quad J\left(\left(E_{i}^{*}\right)_{U}\right)=F \subset G^{*}, \quad Z^{\prime}=\left(K_{i}\right)_{U}^{+}, \quad Z=J\left(K_{i}^{+}\right)_{U} \subset F .
$$

Außerdem bezeichnen wir das Element $J\left(f_{i}\right)_{U}$ ebenfalls als $\left(f_{i}\right)_{U}$.

In den 'Theoremen 1 und 2 werden halbgeordnete Räume $E$ ' betrachtet. Dabei werden im Unterschied zum Ultraproduktfall folgende Bezeichnungen verwendet:

$J$ ist die kanonische Einbettung von $E$ in $E^{* *}, J E=F$,

$$
E^{*}=G, \quad E^{* *}=G^{*}, \quad J K=Z \subset F, \quad K^{++}=Z^{\prime} \subset G^{*} \text {. }
$$


Das Produkt $E^{n}$ von $n$ Banachräumen $E$ ist mit der Norm

$$
\cdot\left\|\left(x_{1} ; \ldots, x_{n}\right)\right\|=\left(\Sigma\left\|x_{j}\right\|^{2}\right)^{1 / 2}
$$

wieder ein Banachraum. Das Produkt der Dualräume $\left(E^{*}\right)^{n}$ wird mit dem Dualraum des Produktes $\left(E^{n}\right)^{*}$ identifiziert, wenn man setzt

$$
\left(f_{1}, \ldots, f_{n}\right)\left(\left(x_{1}, \ldots, x_{n}\right)\right)=\sum f_{j}\left(x_{j}\right)
$$

Durch die Formel

$$
\left(x_{1 i}, \ldots, x_{n i}\right)_{U}=\left(\left(x_{1 i}\right)_{U}, \ldots,\left(x_{n i}\right)_{U}\right)
$$

wird $\left(E_{i}{ }^{n}\right)_{U}$ mit $\left(\left(E_{i}\right)_{U}\right)^{n}$ identiffiziert. Diese Identifizierungen werden wir ohné weiteren Hinweis verwenden.

\section{Formulierung der Resultate}

In diesen Abschnitt wérden die Theoreme über lokale Reflexivität bzw, iiber lokale Dualität von Ultraprodukten für halbgeordnete Banachräume und für Banachverbände formuliert. Die folgenden Abschnitte beinhalten die Beweise für diese Theoreme.

Theorem 1: Sei $E$ ein Banachraum, $K \subset E$ ein Kegel, $\varepsilon>0$, und'seien $L \subset E^{* *}$ -

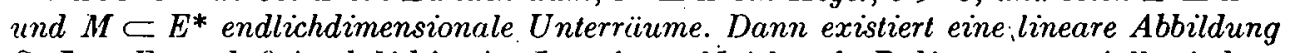
$S: L \rightarrow E$, so daß für beliebige $f \in L$ und $x \in M$ folgende Bedingungen erfüllt sind:

1. $(1-\varepsilon)\|f\| \leqq\|S f\| \leqq(1+\varepsilon)\|f\|$.

2. $x(S f)=f(x)$.

3. $d(S f, K) \leqq d\left(f, K^{++}\right)+\varepsilon\|f\|$.

4. Wenn $f \in J(E)$ ist, dann gilt $J S f=f$.

Theorem 2: Sei $E$ ein Banachverband, $L \subset E^{* *}$ ein endlichdimensionaler Unterverband, $\dot{M} \subset E^{*}$ ein endlichdimensionaler Unterràum und $\varepsilon>0$. Dann existiert ein linearer Operator $S: L \rightarrow E$, so daß für beliebige $f, g \in L$ und $x \in M$ folgende Bedingungen erfülll sind:

1. $(1-\varepsilon)\|f\| \leqq\|S f\| \leqq(1+\varepsilon)\|f\|$.

2. $|x(S f)-f(x)| \leqq \varepsilon\|f\|\|x\|$.

3. $S(f \vee g)=(S f) \vee(S g)$.

4. Wenn $f \in J K$ ist, dann gilt $J S f \leqq f$.

Hat E ordnungsstetige Norm, so kunn zúsützlich erreicht werden:

5. Wenn $f \in J E \cap L$ ist, dann gilt $\|J S f-f\| \leqq \varepsilon\|f\|$.

Theorem 3: Sei $E_{i}$ eine Familie von halbgeordneten Banachrüumen mit Positivitätskegeln $K_{i} \subset E_{i}$ und sei $U$ ein Ultrafilter auf der Indexmenge I. Seien $L \subset\left(E_{i}\right)_{U}{ }^{*}$ und $M \subset\left(E_{i}\right)_{y}$ separáble Unterrïume. Dann existieri ein linearer Operator $S: L \rightarrow\left(E_{i}{ }^{*}\right)_{\dot{U}}$, so da $\beta$ für beliebige $f \in L$ und $x \in M$ folgendes gill:

1. $\mid \mathrm{j} S f\|=\| f \|$.

2. $J S f(x)=f(x)$.

3. $d\left(S f,\left(K_{i}^{+}\right)_{U}\right) \leqq d\left(f,\left(K_{i}\right)_{U^{*}}\right)$.

4. Wenn $f^{\prime} \in J\left(E_{i}^{*}\right)_{U}$ ist, dann gilt $J S i=f$.

Außerdem ist $J\left(K_{i}{ }^{+}\right)_{U}=\left(K_{i}\right)_{U}{ }^{+} \cap J\left(E_{i}{ }^{*}\right)_{U}$, und folglich ist $\left(K_{i}{ }^{+}\right)_{U}$ abgeschlossen. ' . 
Theorem 4: Sei $E_{i}$ eine Familie von Banachverbänden, $U$ ein Dltrafilter. uuf der Indexmenge $I, L \subset\left(E_{i}\right)_{U}{ }^{*}$ ein endlichdimensionaler Unterverband und $M \subset\left(E_{i}\right)_{U}$ ein separabler Unterraum. Dann existiert ein linearer Operator $S: L \rightarrow\left(E_{i}^{*}\right)_{U}$, so daß für beliebige $f, g \in L$ und $x \in M$ gilt:

1. $\|S f\| \doteq\|f\|$.

2. $J S f(x)=f(x)$.

3. $(S f) \vee(S g)=S(f \vee g)$.

4. Wenn $f \in J\left(K_{i}{ }^{+}\right)_{U}$ ist $\left(d . h . f \in J\left(E_{i}^{*}\right)_{:}:\right.$und $\left.f \geqq 0\right)$, dann gilt $(S f) \wedge(f-S f)=0$.

Bemerkungen: Theorem 1 ohne Behauptung 3 ist das Prinzip der lokalen Reflexivität in der Fassung von W. B. Jornson, H., P. Rosentral und M. ZiPPIN [7]. Theorem 2 ohne Behauptung 4 wurde von S. J. BERNaU [1] veröffentlicht. Theorem 3 unterscheidet sich von der Formulierung der lokalen Dualität von Ultraprodukten durch S. HennRICn [6] dadurch, daß Behauptung 3 hinzugekommen ist und daß die abzählbare Unvollständigkeit des Ultrafilters für den Beweis nicht mehr benötigt wird.

\section{.4. Endliche Charakterisierung von Eigenschaiten endlichdimensionaler Operatoren}

Zur Definition linearer Operatoren werden,Gleichungen $T f_{j}=g_{j}$ Verwendung finden, wobei $\left(f_{j}\right)_{j-1}^{m}$ eine vorher festgelegte endliche oder unendliche Folge in $G^{*}$ sein wird, während $\left(g_{j}\right)_{j-1}^{m}$ éine Folge sein wird, die bestimimten Bedingungen genïgt. Bei der Formulierung dieser Bedingungen spielen die im folgenden definierten Mengen logischer Formeln eine Rolle. Die auftretenden.Parameter $a_{\text {; }}$ aus dem Zahlenkörper $\mathbf{R}$ oder $\mathbf{C}, c \in[0, \infty), x_{j} \in G$ und $f \in F$ sollen dabei jeweils die angegebene Menge durchlaufen, wobei aber für jede Formel mindestens eins und höchstens endlich viele ' der Elemente $x_{j}$ bzw. Zahlen $a_{j}$ von Null verschieden sind. Es sei nun

$P_{1}$ die Menge der Formeln

$$
\left\|\sum_{j=1}^{m} a_{j} g_{j}\right\| \leqq c \text { und }\left|\sum_{j=1}^{m} g_{j}\left(x_{j}\right)-b\right| \leqq c,
$$

$\mathbf{P}_{2}$ die Menge der Formeln

$$
d\left(\sum_{j=1}^{m} a_{j} g_{j}+f, Z^{\prime}\right) \leqq c,
$$

$P_{3}$ die Menge der Formeln

$$
\left\|\sum_{j=1}^{m} a_{j} g_{j}+f\right\| \leqq c
$$

und $P_{4}$ die Menge der Formeln .

$g_{j} \in Z^{\prime}$ (d. h., für jedes $j$ wird eine Formel erhalten).

Wir setzen $P=P_{1} \cup P_{.2} \cup P_{3} \cup P_{4}$.

Für $Q \subset P$ bedeutet $\left(g_{j}\right) \models Q$, daß $\left(g_{j}\right)_{j=1}^{m}$ eine Folge von Elementen von $G$ ist, die jede Formel aus $Q$ erfïllt. Fiir logische Formeln $p \in P$ werden außerdem noch Approximationen betrachtet. Und zwar bezeichnen wir für $\varepsilon \geqq 0$ mit $p(\varepsilon)$ die Formel, die man aus $p$ erhält, indem man $c$ durch $c+\varepsilon$ und $Z^{\prime}$ durch $Z$ ersetzt und außerdem $g_{j} \in F$ (für alle $j$ ) fordert. Ist $G=\left(E_{i}\right)_{U}$, so hat jeder Parameter $x_{j}$ bzw. $f$, der in $p$ 
eingeht, eine Darstellung $\left(x_{j i}\right)_{U}$ bzw. $\left(f_{i}\right)_{v}$, wobei (für jedes feste $p$ ) die Elemente $x_{j i} \in E_{i}$ und $f_{i} \in E_{i}{ }^{*}$ für das weitere festgehalten werden sollen. Die Formel $p(\varepsilon, i)$ erhält man in diesen Falle aus der Formel $p$, indem man $c$ durch $c+\varepsilon, Z^{\prime} \operatorname{durch} K_{i^{*}}^{+}$, $f$ durch $f_{i}$ und $x_{j}$ durch $x_{j i}$ ersetzt und außerdem noch $g_{j} \in E_{i}{ }^{*}$ (für alle $j$ ) verlangt. Ist $Q \subset P$, so sei $Q(\varepsilon)=\{p(\varepsilon): p \in Q\}$ und $Q(\varepsilon, i)=\{p(\varepsilon, i): p \in Q\}$. Ist $Q$ eine Menge approximierter Formeln, so bedeutet $\left(g_{j}\right) \models Q$, da $B\left(g_{j}\right)_{j-i}^{m}$ eine Folge von Elementen des entsprechenden Raumes ist, die jede Formel aus $Q$ erfüllt.

Lemma 1: Sei $\theta>0$, seien $L \subset G^{*}$ und $M \subset G$ endlichdimensionale Teilräume und sei $f_{1}, \ldots, f_{m}$ eine Basis von $L$. Dann existieren eine positive Zahl $\delta$ und endliche Teilmengen $Q \subset P_{1}$ und $R \subset P_{2}$, so daß folgendes gilt:

1. $\left(f_{j}\right) \models Q \cup R$.

2. Aus $\left(g_{j}\right) \models Q(\delta)$ folgt für den durch $T f_{j}=g_{j}$ auf $L$ definierten linearen Operator und für beliebige $f \in L$ und $x \in M$

$$
\begin{aligned}
& (1-\theta)\|f\| \leqq\|T f\| \leqq(1+\theta)\|f\|, \\
& |(f-T f)(x)| \leqq \theta\|f\|\|x\| .
\end{aligned}
$$

3. Aus $\left(g_{i}\right) \models Q(\delta)$ ن $R(\delta)$ folgt für den in Behauptung 2 definierten linearen Operaior $T$ ' und für beliebiges $f \in L$

$$
d(T f, Z) \leqq d\left(f, \dot{Z}^{\prime}\right)+\theta\|f\| \text {. }
$$

Beweis: Sei vorerst $0<\delta<1$ beliebig. Sind $f=\sum_{j=1}^{m} a_{j} f_{j}$ und $x \in G$ beliebig, aber fest vorgegeben, so werden die folgenden Formeln durch $\left(f_{j}\right)_{j-1}^{m}$ erfüllt und liègen in $P_{1}$ bzw. $P_{2}$ :

$$
\left\|\sum_{j=1}^{m} a_{j} g_{j}\right\| \leqq\|f\|, \quad\left|\sum a_{j} g_{j}(x)-f(x)\right| \leqq 0, d\left(\sum a_{j} g_{j}, Z^{\prime}\right) \leqq d\left(f, Z^{\prime}\right) .
$$

Ist $T$ der in Behauptung 2 definierte lineare Operator, so sind diese Formeln äquivalent zu

$$
\|T f\| \leqq\|f\|, \quad\left|\left(T^{\prime} h\right)(x)-h(x)\right| \leqq 0, \quad d\left(T^{\prime} f, Z^{\prime}\right) \leqq d\left(f, Z^{\prime}\right) .
$$

Ist nun $A$ ein endliches $\delta$-Netz $\operatorname{der}$ Sphäre $\{f \in L:\|f\|=1\}$, und bestimmt man eine endliche Menge $B \subset G$ so, daß $B$ ein endliches $\delta$-Netz der Sphäre $\{x \in M:\|x\|=1\}$ ist und außerdem für jedes $f \in A$ ein $x$ mit $\|x\|=1$ und $|f(x)| \geqq 1-\delta$ enthält, so erhält man die endlichen Formelmengen $Q \subset P_{1}$ und $R \subset P_{2}$, indem in den oben angegebenen Formeln $/$ die Menge $A$ und $x$,die Menge $B$ durchläuft.

Sei $f \in L,\|f\|=1$ und sei $x \in M,\|x\|=1$. Es gibt Darstellungen,

$$
\begin{aligned}
& f=\sum_{s=0}^{\infty} \lambda_{s} h_{s} \text { mit }\left|\lambda_{s}\right| \leqq \delta^{s} \text { und } h_{s} \in A, \\
& x=\sum_{s=0}^{\infty} \mu_{s} y_{s} \text { mit }\left|\mu_{s}\right| \leqq \delta^{s} \text { und } y_{s} \in B,
\end{aligned}
$$

sowie Elemente $h \in A$ und $y \in \vec{B}$ mit $\|f-h\| \leqq \dot{\delta},\|y\|=1$ und $|h(y)| \geqq 1-\delta$. Aus $\left(g_{i}\right) \models Q(\delta)$ und $T f_{j}=g_{j}$ folgt dann

$$
\|T f\| \leqq \sum\left|\lambda_{s}\right|\left\|T h_{s}\right\| \leqq(1+\delta) \sum_{s=0}^{\infty} \delta^{s}=(1+\delta) /(1-\delta),
$$




$$
\begin{aligned}
& || T f|| \geqq|T f(y)| \geqq|(T(f-h))(y)+(T h)(y)| \geqq 1-2 \delta-\delta(1+\delta) /(1-\delta), \\
& |T f(x)-f(x)|=\left|\sum_{r, s=0}^{\infty} \lambda_{s} \mu_{r}\left(T h_{s}-h_{s}\right)\left(y_{r}\right)\right| \leqq \delta(1-\delta)^{-2} \cdot
\end{aligned}
$$

Ist auBerdem $\left(g_{j}\right) \models R(\delta)$ erfüllt, so folgt

$$
\begin{aligned}
d\left(T^{\prime} f, Z\right) & \leqq d(T h, Z)+\|T(f-h)\|^{\prime} \leqq d\left(h, Z^{\prime}\right)+\delta+\|T\|\|f-h\| \\
& \leqq d\left(f, Z^{\prime}\right)+\delta+\delta+\delta(1+\delta) /(1-\delta) .
\end{aligned}
$$

Ist $\delta$ genügend klein, so sind also alle Behauptungen von Lemma 1 erfüllt

Lemma 2: Sei $G$ ein Banachverband und $\delta>0$. Wenn $f_{1}, \ldots, f_{m} \in G^{*}, f_{j} \wedge f_{k}=0$ $(k \neq j)$ und $0 \leqq x \in G$ ist, dann existieren $x_{1}, \ldots, x_{m} \in G$, so da $\beta x_{j} \geqq 0, x=x_{1}+\cdots$ $+x_{m}$ und $f_{j}\left(\dot{x}_{k}\right)<\delta(k \neq j)$ gilt.

Dieses Lemma ist ein Spezialfall von Lemma 7 in [1] und besitzt einen einfachen Induktionsbeweis.

Lem ma 3: Séien $G$ und $F \subset G^{*}$ Banuchverbände, $L \subset G^{*}$.ein endlichdimensionaler Unterverband und $f_{1}, \ldots, f_{m}$ eine Basis aus Atomen für L. Seien weiterhin ein $0>0$ und eine endliche Menge $Q \subset P_{1}$ mit $\left(f_{j}\right) \vDash Q$ gegeben. Dann existiert eine Zahl $\delta \in(0,1)$ und eine endliche Menge $R \subset P_{1}$, so daß $\left(f_{i}\right) \models R$ gill, und so daß aus. $\left(h_{j}\right) \models R(\delta)$ und aus

$$
d\left(g_{i},\left[\left(h_{j}-\delta^{-1 / 2} \sum_{k \neq j} h_{k}\right)^{+},\left(1+\delta^{1 / 2}\right) h_{j}\right]\right) \leqq \delta
$$

folgt $\left(g_{j}\right) \models Q(\theta)$.

Beweis: Sei $\delta \in(0,1)$ vorerst beliebig und sei

$$
e_{j} \in\left[\left(h_{j}-\delta^{-1 / 2} \sum_{k \neq j} h_{k}\right)^{+},\left(1+\delta^{1 / 2}\right) h_{j}\right] \text {. }
$$

Angenommen, es gilt $\left\|g_{j}-e_{j}\right\| \leqq \delta$. Es reicht aus, das Lemma für den Fall einer einelementigen Menge $Q=\{p\}$ zu beweisen, weil man im allgemeinen Fall die für die einzelnen Formeln aus $Q$ erhaltenen Mengen $R$ vereinigen und das mininale $\delta$ nehmen kann. $\Psi \mathrm{m}$ weiteren Beweis werden zwei Fälle unterschieden.

1. Fall: Sei $p=\left(\left\|\sum a_{j} g_{j}\right\| \leqq c\right)$.

Fs gilt $\left\|\Sigma\left|a_{j}\right| f_{j}\right\|=\left\|\Sigma a_{j} f_{j}\right\| \leqq c^{\prime}$. Gehört die Formel $\left(\left\|\Sigma\left|a_{j}\right| g_{j}\right\| \leqq c\right)$ zu $R$, so folgt aus $\left(h_{j}\right) \models R(\delta)$,

$$
\begin{aligned}
\left\|\sum a_{j} g_{j}\right\| & \leqq \delta \sum\left|a_{j}\right|+\left\|\sum a_{j} e_{j}\right\| \\
\cdot & \leqq \delta \sum\left|a_{j}\right|+\left(1+\delta^{1 / 2}\right)\left\|\Sigma\left|a_{j}\right| h_{j}\right\| \\
& \leqq \delta \sum\left|a_{j}\right|+\left(1+\delta^{1 / 2}\right)(c+\delta) .
\end{aligned}
$$

Für genügend kleines $\delta$ folgt also die Behauptung.

2. Full: Sei $p=\left(\left|\sum g_{j}\left(x_{j}\right)-b\right| \leqq c\right)$.

. Wendet man auf den positiven Teil $x_{j}^{+}$und den negativen Teil $x_{j}^{-}$von $x_{j}$ jeweils Lemma 2 an, so findet man nichtnegative Elemente $x_{j k}^{+}$und $x_{j k}^{-}$, so da $x_{j}^{ \pm}=\sum_{k=1}^{m} x_{j k}^{ \pm}$
und (falls $k \neq l$ ist) $f_{l}^{\prime}\left(x_{j k}^{ \pm}\right)<\delta$ gilt. Sind nun die Formieln

$$
\left(\left|g_{l}\left(x_{j k}^{ \pm}\right)-f_{l}\left(x_{j k}^{ \pm}\right)\right| \leqq 0 \quad(j, k, l \in\{1, \ldots, m\})\right.
$$


in $R$ enthalten, so folgt aus $\left(h_{j}\right) \models R(\delta)$ für festes $j$ und festes $k$ :

$$
\begin{aligned}
\left(1+\delta^{1 / 2}\right) h_{j}\left(x_{j k}^{ \pm}\right) & \geqq e_{j}\left(x_{j k}^{ \pm}\right) \geqq\left(h_{j}-\delta^{-1 / 2} \sum_{l \neq j} h_{l}\right)^{+}\left(x_{j k}^{ \pm}\right) \\
& \geqq h_{j}\left(x_{j k}^{ \pm}\right)-\delta^{-1 / 2} \sum_{l \neq j} h_{i}\left(x_{j k}^{\prime}\right) \geqq h_{j}\left(x_{j k}^{ \pm}\right)-2 \delta^{1 / 2} m .
\end{aligned}
$$

Man erhält weiter:

$$
\begin{aligned}
\left|\left(h_{j}-e_{j}\right)\left(x_{j k}^{ \pm}\right)\right| & \leqq \delta^{1 / 2}\left(2 m+h_{j}\left(x_{j k}^{ \pm}\right)\right) \leqq \delta^{1 / 2}\left(2 m+\left\|f_{j}\right\|\left\|x_{j}\right\|+\delta\right), \\
\left|\left(g_{j}-f_{j}\right)\left(x_{j k}^{ \pm}\right)\right| & \leqq\left|\left(\left(g_{j}-e_{j}\right)+\left(e_{i}-h_{j}\right)+\left(h_{j}-f_{j}\right)\right)\left(x_{j k}^{ \pm}\right)\right| \\
& \leqq \delta\left\|x_{j}\right\|+\delta^{1 / 2}\left(2 m+\left\|f_{j}\right\|\left\|x_{j}\right\|+\delta\right)+\delta .
\end{aligned}
$$

Fiir genügend kleines $\delta$ folgt daraus.

$$
\begin{aligned}
\left|\sum g_{j}\left(x_{j}\right)-b\right| & =\left|\sum_{j, k} g_{j}\left(x_{j k}^{+}-x_{j k}\right)-b\right| \\
& \leqq\left|\sum_{j, k} f_{j}\left(x_{j k}^{+}-x_{j k}^{\bar{j}}\right)-b\right|+0 \leqq c+\theta
\end{aligned}
$$

Bemerkung: Es seien Zahlen $\lambda_{k} \in\{0,1\}$ mit $\lambda_{j}=1$ ( $j$ wird festgehalten) und ein Flement $e$ mit $\left\|\sum_{k=1}^{m} \lambda_{k} h_{k}-e\right\| \leqq \delta$ gegeben. Sind die $h_{j}$ positiv und ist $\eta \in\left[\delta^{1 / 2}\right.$, $\left.\delta^{-1 / 2}\right]$, so gilt $(4 \hat{)}$, z. B., wenn

oder

$$
g_{j} \doteq\left(h_{j} \dot{-} \eta \sum_{k \neq j} h_{k}\right)^{+} \wedge e
$$

$$
g_{j}=\sup _{l \in \mathbf{N}}\left(l\left(h_{j}-\delta^{-1 / 2} \sum_{k \neq j^{*}} h_{k}\right)^{+}\right) \wedge \sum \lambda_{k} h_{k}
$$

. ist. Um dies für die erste Formel zu beweisen, setzen wir $d \doteq\left(h_{j}-\eta \sum_{k \neq j} h_{k}\right)^{+}$. Es folgt $0 \leqq d-g_{j}=d-d \wedge e=(d-e)^{+}$. $\left(\sum \lambda_{\lambda_{k}} h_{k}-e\right)^{+}, \quad$ also gilt " $\left\|d-g_{j}\right\|$ $\leqq\left\|\sum \lambda_{k} h_{k}-e\right\| \leqq \delta$.

Für die żweite Formel benutzen wir, da 3 für beliebige positive Elemente $d, f \in G^{*}$ und für $c \in(0,1)$ gilt

$$
\begin{aligned}
\left(m\left(d-c^{-1} f\right)^{+}\right) \wedge(d+f) & \leqq\left(m c^{-1}(c d-f)^{+}\right) \wedge\left((1+c) d+(f-c d)^{+}\right) \\
& \leqq(1+c) d .
\end{aligned}
$$

Es folgt fiir jede natiirliche Zahl $l$ :

$$
\left(h_{j}-\delta^{-1 / 2} \sum_{k \neq j} h_{k}\right)^{+} \leqq\left(l\left(h_{j}-\delta^{-1 / 2} \sum_{k \neq j} h_{k}\right)^{+}\right) \wedge \sum_{k=1}^{m} \lambda_{k} h_{k} \leqq\left(1+\delta^{1 / 2}\right) h_{j} .
$$

\section{Ein Trennungssat\%}

Der folgende 'Trennungssatz für eine Anzahl konvexer Mengen ist das wesentliche Hilfsmittel zum Beweis der Theoreme. Er wird hier nur für Banachräume benötigt, wird aber für beliebige lokalkonvexe Räume bewiesen. Varianten des Satzes sind schon bekannt. So ist' das Lemma von A. JA. Dubowizki und A. A. Mrluutis (vgl. z. B. [15]) ein Spezialfall von Lemma 4a).

Lenma 4: a) Seien $C_{1}, \ldots, C_{r}$ konvexe Teilmengen von $E$ mil nichtleerem Inneren und $C_{0} \subset E$ eine nichtleere konvexe Menge. Ist kein Punkt von $C_{0}$ zugleich innerer 
Punkt von $C_{1} \cap \ldots \cap C_{r}$, so existieren reelle Zahlen $b_{0}, \ldots, b_{r}$ und Elemente $f_{0}, \ldots, f_{r}$ des Dualraumes $E^{*}$, von denen wenigstens eins von $N$ ull verschieden ist, so da $\beta$

$$
\sum f_{s}=0, \quad \Sigma b_{s}=0 \text {, und } \operatorname{Re} f_{s}(x) \geqq b_{s} \text { im Falle } x \in C_{s}
$$

gilt.

b) Sind $C_{s}(s=0,1, \ldots, r)$ nichtleere $\sigma\left(E^{*}, E\right)$-abgeschlossene ,konvexe T'eilmengen des Dualraumes $E^{*}$ mit leerem Durchschnitt und ist $C_{0} \sigma\left(E^{*}, E\right)$-kompakt, so existieren. Elemente $x_{0}, \ldots, x_{r}$ von $E$ und reelle Zahlen $b_{0}, \ldots, b_{r}$, so daß

$$
\sum x_{s}=0, \sum b_{s}>0,- \text { und } \operatorname{Re} f\left(x_{s}\right) \geqq b_{s} \text { im Falle } f \in C_{s}
$$

gilt.

Beweis: Es reicht aus, das Lemma für reelle lokalkonvexe Räume zu beweisen, denn wenn $E$ ein komplexer lokalkonvexer Raum ist, dann kann $E^{*}$ mit dem Dualraum zum zu $E$ assoziierten reellen Raum identifiziert werden. Dem Funktional $f$ entspricht dabei das reell-lineare Funktional $x \rightarrow \operatorname{Re} f(x)$ (vgl: [12]).

a) 1. Schritt: Hat ein endlicher Durchschnitt von Kegeln $\cap K_{s}$ innere Punkte, so gilt $\cap \bar{K}_{s}=\overline{\cap K_{g}}$. (Mit $\bar{C}$ wird der Abschluß einer Menge $C$, bezeichnet.) Sind-nämlich eine Nullumgebung $B$ und ein $x_{0}$ so gewählt, da $B x_{0}+B \subset \cap K_{s}$ gilt, so folgt für beliebiges $\varepsilon>0$

$$
\cap \bar{K}_{s} \subset n\left(K_{s}+\varepsilon B\right) \subset \cap\left(K_{s}+K_{s}-\varepsilon x_{0}\right)=-\varepsilon x_{0}+n K_{s} .
$$

2. Schritt: Hat cin endlicher Durchschnitt vou Kegeln innere Punkte, so gilt $\left(\cap K_{s}\right)^{+}=\sum K_{s}{ }^{+}$. In der Tat, nach einer Schlußfolgerung aus dem Bipolarensatz (ंvgl. [12]) ist $\sum K_{s}{ }^{+}$in $\left(\cap \bar{K}_{s}\right)^{+}=\left(\overline{\cap K_{s}}\right)^{+}=\left(\cap K_{s}\right)^{+}$dicht bezüglich der schwachen Topologie $\sigma\left(E^{*}, E\right)$. Konvergiert aber $h_{i}=\sum h_{s i}\left(h_{s i} \in K_{s}{ }^{+}\right)$bezüglich eines Ultrafilters $U$ in $\operatorname{der} \sigma\left(E^{*}, E\right)$-Topologie gegen $h \in E^{*}$, so gilt für Elemente $x$ einer in $\cap K_{s}$ enthaltenen offenen Menge $0 \leqq h_{s i}(x) \leqq h_{i}(x)$. Deshalb. existiert $\sigma\left(E^{*}, E\right)$-lim $h_{s i}$ $=h_{s} \in K_{s}^{+}$, und es gilt $h=\sum h_{s}$. Also ist $\sum K_{s}{ }^{+}$bereits abgeschlossen.

3. Schritt: Der von $C_{s} \times 1$ in $E \times \mathbf{R}$ erzeugte Kegel werde mit $K_{s}$ bezcichnet. Es cxistiert eine eindeutig bestimmte Zahl $n$, so $\operatorname{da} \beta \stackrel{\Upsilon}{r}_{s=n+1}^{r} K_{s}$ innere Punkte enthält, während das Innere von $\stackrel{r}{\bigcap} K_{s}$ leer ist. Die konvexen. Mengen $K_{n}$ und $\bigcap^{r} K_{s}$ lassen' sich nun durch eine Hyperebene trennen, d. h., es existiert ein $h \in\left(E^{n+1} \times \mathbf{R}\right)^{*}$ mit $h \neq 0, h \in K_{n}^{+}$und $-h \in\left(\bigcap_{n+1}^{r} K_{s}\right)^{+}=\sum_{n+1}^{r} K_{s}{ }^{+}$. Also ist $-h=\sum_{n+1}^{r} h_{s}$ mit $h_{s} \in K_{s}{ }^{+}$. Verwendet man dic Identifizierung $(E \times \mathbf{R})^{*}=E^{*} \times \mathbf{R}$ und setzt $: f_{s}=0$ und $b_{s}=0$ fiir $s<n,\left(f_{n},-b_{n}\right)=h$ und $\left(f_{s},-b_{s}\right)=h_{s}$ fiir $s>n$, so sind alle Behauptungen von Lemma 4 a) erfüllt.

b) Jetzt sei für $s \geqq 1 \mathrm{mit}^{*} K_{s}$ der von $C_{s} \times 1^{\prime}$ in $E^{*} \times \mathbf{R}=(E \times \mathbf{R})^{*}$ erzeugte $w^{*}$-abgeschlossene konvexe Kegel bèzeichnet: AuBerdem sei $K_{0}=C_{0} \times 1$. Elemente aus $\cap K_{s}$ haben notwendigerweise die Gestalt $(f, 1)$ mit $f \epsilon \cap C_{s}$. Also lassen sich die Mengen $K_{0}$ und $\bigcap_{s=1}^{r} K_{s}$ durch eine $\sigma\left(E^{*}, E^{\prime}\right)$-abgeschlossene Hyperebene streng trennen - es existiert ein $y \in E \times \mathbf{R}$ mit

$$
\sup \left\{h(y): h \in K_{0}\right\}<0=\inf \left\{h(y): h \in \check{\cap}_{s=1}^{r} K_{s}\right\} .
$$


Nun ist $\sum_{s=1}^{r}\left(K_{s}\right)_{+}$auf Grund der bereits erwähnten Schlußfolgerung aus dem Bipolarensatz schwach dicht in $\left(\bigcap_{s=1}^{r} K_{s}\right)_{+}$. Konvergiert $y_{i} \in \sum_{s=1}^{r}\left(K_{s}\right)_{+}$, bezüglich eines Ultrafilters $U$ schwach gegen $y$, so folgt aus der Konipaktheit von $K_{0}$ für fast alle $i$

$$
\sup \left\{h\left(y_{i}\right): h \in K_{0}\right\}<0 \text { : }
$$

Man kann deshalb (nachdem eventuell $y$ durch ein geeignetes $y_{i}$ ersetzt wurde) voraussetzen $y=\sum_{s=1}^{r} y_{s}=\sum_{s=1}^{r}\left(x_{s},-b_{s}\right)$, wobei $y_{s}=\left(x_{s},-b_{s}\right) \in\left(K_{s}\right)_{+}$ist. Setzt. man noch

$$
\left(x_{0},-b_{0}\right)=-y_{0}-\left(0, \sup \left\{h(y): h \in K_{0}\right\}\right)
$$

so sind alle Behauptungen von Lemma 4 b) erfüllt

Bemerkung: Lemma 4 läßt sich umkehren. Sind z. B.,konvexe Mengen $C_{0}, \ldots, C_{r}$ in $E$, reelle Zahlen $b_{0}, \ldots, b_{r}$ und Funktionale $f_{0}, \ldots, f_{r}$ gegeben, und ist $(5)$ erfüllt, so enthält $C_{0}$ keinen inneren Punkt von $C_{1} \cap \ldots \cap C_{r}$. Wäre $x$ ein solcher Punkt, so würde folgen $f_{s}(x) \geqq b_{s}$, und wenigstens eine dieser Ungleichungen wäre echt, also wäre $0=\sum f_{s}(x)>\sum b_{s}=0$.

\section{Beweis der Theoreme 1 und 2}

Im folgenden Lemma 5 und beim Beweis der Theoreme 1 und 2 werden die dafür eingefiihrten Bezeichnungen $G=E^{*}, Z=J K$ usw. verwendet.

Lemma 5: Seien eine endliche Menge von Formeln $Q \subset P$ und eine Folge $\left(f_{i}\right)_{j-1}^{m}$ in $G^{*}$ so gegeben, $\dot{d a} \beta\left(\dot{f}_{j}\right) \models Q$ gilt, und sei $\delta>0$. Dann gibt es eine Folge $\left(g_{j}\right)$ mit $\left(g_{j}\right) \models Q(\delta)$.

Beweis: Da nur endlich viele $g_{j}$ in Formeln $p \in Q$ eingehen, können wir voraussetzen, da $m<\infty$ ist. Es reicht nun aus, zu zeigen, $\mathrm{da} B$ folgende Teilmengen von $-E^{m}$ nichtleeren Durchschnitt haben:

$$
\begin{aligned}
& C_{0}=\left\{\left(g_{1}, \ldots, g_{m}\right):\right. g_{j} \in K \text { für alle diejenigen } j, \text { für die die Formel }\left(g_{j} \in Z^{\prime}\right) \\
&\text { zu } Q \text { gehört }\}, \\
& C_{p}=\left\{\left(g_{1}, \ldots, g_{m}\right):\left(J g_{j}\right) \models p(\delta)\right\} \quad\left(p \in Q \backslash P_{4}\right) .
\end{aligned}
$$

Für $p \in Q \backslash P_{\text {qgehört }}\left(f_{1}, \ldots, f_{m}\right)$ zum $w^{*}$-Abschluß von $\left\{\left(g_{1}, \ldots, g_{m}\right):\left(g_{j}\right) \models p(\delta / 2)\right\}$. Es ist leicht zu sehen, daß die Mengen $C_{p}$ innere Punkte enthalten, und daß $\left(f_{1}, \ldots, f_{m}\right)$ innerer Punkt vom $w^{*}$-Abschlu $\beta$ von $J C_{p}$ ist. Außerdem gehört $\left(f_{1}, \ldots, f_{m}\right)$ zum $w^{*}$-Abschluß von $J C_{0}$. Wäre der Durchschnitt der betrachteten Mengen leer, so würde nit Hilfe von Lenma 4a) folgen, daß kein Punkt gleichzeitig zum $w^{*}$-Abschlu $B_{\text {, von }} C_{0}$ und zum Inneren des $w^{*}$-Abschlusses jeder der Mengen $C_{p}\left(p \in Q \backslash P_{4}\right)$ gehört. Àlso ist Lemma 5 bewiesen

Bemerkung: Als SchluBfolgerung aus Lemma 5 erhält nian, daß

$$
\left\{x \in E^{* *}:\|x\| \leqq 1, x \in K^{++}\right\}
$$

$\operatorname{der} w^{*}$-Abschlu $\beta$ von $J(\{x \in E:\|x\| \leqq 1, x \in K\})$ ist. Lemma 5 in [1] beińhaltet dieselbe Aușsage unter zusätzlichen Voraussetzungen. 
Beweis von Theorem 1: Sei $f_{1}, \ldots, f_{m}$ eine Basis von $L$, so da $B f_{n+1}, \ldots, f_{m}$ eine Basis von $J E \cap L$ ist, und sei $0>0$. Seien ferner $Q \subset P_{1}, R \subset P_{2}$ und $\delta>0$. so definiert, daß die Behauptungen von Lenma 1 gelten. Aus Lemma 5 folgt die Existenz einer Folge $\left(g_{j}\right)$ mit $\left(g_{j}\right) \models(Q \cup R)(\delta)$, so da $B$ außerdem noch $\left\|g_{j}-f\right\| \leqq \delta$ für $n<j \leqq m$ erfiillt ist. Schließlich sei $T_{1}: E \rightarrow E / M_{+}$die kanonische Quotientenabbildung und $T_{2}: E^{* *} / M^{+} \rightarrow E$ solch eine lineare Abbildung, da $B T_{1} T_{2}$ die kanonische Isometrie von $E^{* *} / M^{+}$anf $E / M_{+}$ist, und sei $T^{\prime}: L \rightarrow F$ durch $T f_{j}=g_{j}$ und $S: L \rightarrow E$ durch

$$
S f_{j}= \begin{cases}J^{-1} g_{j}+T_{2} T_{1}{ }^{* *}\left(f_{j}-g_{j}\right) & \text { für } 1 \leqq j \leqq n \\ J^{-1} f_{j} & \text { für } n<j \leqq m\end{cases}
$$

definiert. Dánn gilt die Behauptung 4 von Théorem 1, während die Behauptung 2 aus $J S f-f \in \operatorname{Ker} T_{1}{ }^{* *}$ fólgt. Ist $c$ solch cine Konstante, da: $\sum\left|\dot{\lambda}_{j}\right| \leqq c\left\|\sum \lambda_{j} j_{j}\right\|$
gilt, so zieht das

$$
\begin{aligned}
& \left\|(J S-T)\left(\sum \lambda_{j} f_{j}\right)\right\| \\
& \vdots \dot{c}\left\|\sum \lambda_{j} f_{j}\right\|\left(\left\|\dot{T}_{2}\right\| \sum_{1}^{n}\left\|T_{1}{ }^{* *}\left(f_{j}-T f_{j}\right)\right\|+\sum_{n+1}^{m} \delta\right)
\end{aligned}
$$

nach sich. Aus Lemma $1 /(2)$ folgt $\left\|T_{1}{ }^{* *}(f-T f)\right\| \leqq \theta\|f\|$. Man kann $\delta$ und $0<\varepsilon / 2$ so klein wählen, daß $\mid J S-T \|<\varepsilon / 2$ ist, denn $c$ und $\left\|T_{2}\right\|$ hängen nicht von $\delta$ und $\theta$ ab. Die Behauptungen 1 und 3 folgen dann aus Lemma $1 /(1)$ und (3)

Beweis von Theorem 2: Sei $f_{1}, \ldots ; f_{m}$ eine Basis aus Atomen für $L$, wobei $f_{n+1}, \ldots, f_{m}$ bereits zu $F$ gehören. Außerdem. seien $e_{1}, \ldots, e_{r}$ so gewählt, daß sie zusammen mit $f_{n+1}, \ldots, f_{m}$ eine Basis aus Atomen für $L \cap F$ bilden. Es kann dabei zusätzlich erreicht werden, daß jedes Element $e_{s}$ eine Darstellung $\sum_{j=1}^{n} \lambda_{s j} f_{i}$ nit $\lambda_{s j} \in\{0,1\}$ besitzt. Iant Lemma 1 existicrt ein $\theta>0$ und eine endliche Menge $Q \subset P_{1}$, so daß $\left(f_{j}\right) \models Q$ gilt, und daß aus $\left(g_{j}\right) \models Q(\theta), i f_{j}=g_{j}$ und aus $f \in L$, $x \in M$ folgt

$$
(1-\varepsilon)\|f\| \leqq\|T f\| \leqq(1+\varepsilon)\|f\|, \quad\left|\left(f-T^{\prime} f\right)(x)\right| \leqq \varepsilon\|f\|\|x\| .
$$

Entsprechend Lemmia 3 seien $R \subset P$ und $\delta \in(0,1)$ bestimmit. $R^{\prime}$ sei aus $R$ durch Hinzunahme der Formeln

$$
\begin{aligned}
& \left(\left\|g_{j}-f_{j}\right\| \leqq 0\right) \quad \cdot \quad(n<j \leqq \dot{m}) \\
& \left(\left\|\sum \dot{\lambda}_{s j} g_{j}-e_{s}\right\| \leqq 0\right), \quad(1 \leqq s \leqq r) \\
& \left(g_{j} \in Z^{\prime}\right) \quad(1 \leqq j \leqq m)
\end{aligned}
$$

erhalten. Aus Lemma 5 folgt die Existenz einer Folge $h_{j}$ mit $\left(h_{j}\right) \models R^{\prime}(\delta)$. Zur Definition von $g_{j}=J S f_{j}$ werden drei Fälle unterschieden.

1. Fall: Es sei $n<j \leqq m$. Wir setzen

$$
g_{j}=\left(h_{j}-\delta^{1 / 2} \sum_{k \neq j} h_{k}\right)^{+} \wedge f_{j}
$$

2. Fall: Es existiert ein $s$ mit $f_{j} \leqq e_{s}$, d. h. es gibt eine eindeutig bestimmte $Z a h l s$, so da $B$ in der Darstellung $\sum_{l=1}^{n} i_{s l} \dot{f}_{l}$ gilt $\dot{\lambda}_{s j}=1$. Wir setzen

$$
g_{j}=\left(h_{j}-\delta^{-1 / 2} \sum_{k \neq j} h_{k}\right) \wedge e_{s} .
$$


3. Fall: Es sei $j_{j} \wedge\left(\sum e_{s}\right)=0$ und $j \leqq i n$. Wir setzen

$$
g_{j}=\left(h_{j}-\delta^{-1 / 2} \sum_{k \neq j} h_{k}\right)^{+}
$$

Aus der Definition der Elemente $g_{j}$ folgt Behauptung 4. Da Isemma 3/(4) nach der -sich dort anschließenden Bemerkung erfüllt ist, folgen die Behauptungen 1 und 2 aus Lemma 1 und 3 . Ist $c \in(0,1)$ ind sind $d, e \in G^{*}$ beliebige-positive Elemente, so gilt

$$
0 \leqq(d-c e)^{+} \wedge\left(e-c^{-1} d\right)^{+} \leqq c^{-1}\left((d-c e)^{+} \dot{\wedge}(c e-d)^{+}\right)=0 .
$$

Daraus folgt $g_{j} \wedge g_{k}=0$, falls $1 \leqq j \leqq n, 1 \leqq k \leqq m$ und $j \neq k$ gilt. Dies gilt aber auch wenn $n<j<k \leqq m$ ist, denn in diesem . Fall ist $0 \leqq g_{j} \wedge g_{k} \leqq f_{j} \wedge f_{k}=0$. Es - folgt die Behauptung 3. Für $n<j \leqq m$ folgt weiter '

$$
\begin{aligned}
& 0 \leqq f_{i}-g_{i} \leqq f_{j}-\left(h_{j} \wedge f_{j}\right)+\dot{\delta}^{1 / 2} \sum h_{k}=\left(f_{j}-h_{j}\right)^{+}+\delta^{1 / 2} \sum h_{k}, \\
& \left\|f_{j}-J S f_{j}\right\| \leqq \delta+\delta^{1 / 2} \sum\left\|h_{k}\right\| \leqq \delta+\delta^{1 / 2} \sum\left(\left\|f_{k}\right\|+\delta\right) .
\end{aligned}
$$

Es ist möglich, $\delta$ von Anfang an so klein zu wählen, daß aus diesen Ungleichungen für jedes Element $f$ aus der linearen Hülle von $f_{n+1}, \ldots, f_{m}$ folgt $\|J S f-f\| \leqq \varepsilon\|f\|$. Hat $E$ ordnungsstetige Norm, so würde aus $0 \leqq f_{i} \leqq e_{s}$ bereits $f_{j} \in J E$ folgen. Also fällt $F \cap L$ mit der linearen Hülle der Elemente $f_{n+1}, \ldots, f_{m}$ zusammen. Damit ist auch Behauptung 5 bewiesen

\section{Eigenschaften von Ultraprodukten}

Beim Beweis von Lemma 5 wurde wesentlich verwendet, daß $\left(f_{1}, \ldots, f_{m}\right)$ zum w*Abschlu $B$ von $C_{p}$ gehörte. Dies folgte daraus, da $B J K \mathrm{w}^{*}$-dicht in $K^{++}$ist und aus einer analogen Aussage für-Einheitskugeln. Um die Theoreme 3 und 4 zu beweisen, benötigen wir entsprechende Resultate für Ultraprodukte. Diese werden. in den nächsten beiden Lemmas bereitgestellt.

Lemma 6: Sei $x_{1}, \ldots, x_{r}$ eine Basis eines endlichdimensionalen Unterraumes $M \subset\left(E_{i}\right)_{U}$ und seien $x_{s i} \in E_{\mathrm{i}}$ so gewühlt, daß $\left(x_{s i}\right)_{U}=x_{s}$ ist. Dann gill für die durch $T_{i} x_{s}=x_{s i}$ definierten lineuren Operatoren $T_{i}$ von $M$ auf die lineare Hülle von $\left\{x_{s i}: s \in\{1, \ldots, r\}\right\} \subset E_{i}$ :

1. $\left(T_{i} x\right)_{U}=x$.

2. Für fast alle $i$ ist $T_{i}$ invertierbar.

3. $\lim _{v}\left\|T_{i}\right\|=\lim _{v}\left\|T_{i}{ }^{-1}\right\|=1$.

Beweis: Nach Definition von $T_{i}$ gilt Behauptung 1 fïr die Basiselemente von $M$ und folglich auch für beliebiges $x \in M$. Angenommen, eine der Behauptungen 2 oder 3 sei nicht erfüllt. Dann existieren $\varepsilon \in(0,1)$ und Elemente $z_{i} \in M$, so da $B$ für fast alle $i \in I$ entweder $\left\|T_{i} z_{i}\right\|<(1-\varepsilon)\left\|z_{i}\right\|$ oder $\left\|T_{i} z_{i}\right\|>(1+\varepsilon)\left\|z_{i}\right\|$ gilt. Im ersten Fall nehmen wir noch $\left\|z_{i}\right\|=1$ und im zweiten Fall $\left\|T_{i} z_{i}\right\|=1$ an. Verwendet man die Basisdarstellúngen $z_{i}=\sum \lambda_{s i} x_{s}$, so bilden die $\lambda_{s i}$, als Koordinaten einer beschränkten Familie in einem endlichdimensionalen Banachraum, ebenfalls beschränkte Fanilien. Aus

$$
\cdot\left(T_{i}^{\prime} z_{i}\right)_{U}=\Sigma\left(\lim _{U} \lambda_{s i}\right)\left(x_{s i}\right)_{U}=\lim _{U} z_{i}
$$

folgt im Widerspruch zu den gemachten Annahmen $\lim _{U}\left\|z_{i}\right\|=\lim _{U}\left\|T_{i} z_{i}\right\|=1$. Damit ist das Lemma bewiesen 
In den folgenden beiden Lemmas, sowie im Beweis der Theoreme 3 und 4 werden die dazu eingeführten Bezeichnungen $G=\left(E_{i}\right)_{U}, Z=J\left(K_{i}{ }^{+}\right)_{U}$ usw. verwendet.

Lemma 7: Seien eine nalürliche Zahl $m$, ein $p \in P$ und eine Folge $\left(f_{j}\right)_{j=1}^{m}$ so vorgegeben, $d a \beta\left(f_{i}\right) \models\{p\}$ ist. Sei ferner.

$$
C_{i}=\left\{\left(g_{1}, \ldots, g_{m}\right) \in\left(E_{i}^{*}\right)^{m}:\left(g_{j}\right) \models\{p(0, i)\}\right\} .
$$

Dann gehört $\left(f_{1}, \ldots, f_{m}\right)$ zum $w^{*}$-Abschluß von $J\left(C_{i}\right)_{U}$ in $\left(G^{*}\right)^{m}$.

Beweis: Für einen vorerst beliebig gewählten endlichdimensionalen Uinterraum $M \subset\left(E_{i}\right)_{U}$ seien $T_{i}$ die in Lemma 6 definierten Operatoren. Für fast alle $i$ werden auf $T_{i}(M)$ durch

$$
h_{j i}(x)=f_{j}\left(T_{i}^{-1} x\right)
$$

lineare Funktionale $h_{j i}$ definiert. Bestimmt man $g_{j}=\left(g_{j i}\right)_{U}$ so, daß $\left.g_{j i}\right|_{r,(M)}=h_{j i}$ ist, so ist eine $\varepsilon$-Umgebung von $\left(g_{1}, \ldots, g_{m}\right)$ in einer beliebig vorgegebenen $w^{*}$-Umgebung von $\left(f_{1}, \ldots, f_{m}\right)$ enthalten, wenn nur $M$ und $\varepsilon>0$ gecignet gewählt wurden. Deshalb reicht es aus, für $\theta>0$ und für fast alle $i$ gleichmäßig normbeschränkte Fortsetzungen $g_{j i}$ von $h_{j i}$ so zu finden, daß

gilt.

$$
d\left(\left(g_{1 i}, \ldots, g_{m i}\right), C_{i}\right) \leqq \theta
$$

1. Fall: Es sei $p=\left(\left|\sum g_{j}\left(x_{j}\right)-b\right| \leqq c\right)$. In diesem Fall wird $M$ so gewählt, da $x_{j} \in M$ ist. Sind $g_{j i}$ Hahn-Banach-Fortsetzungen von $h_{j i}$, so folgt für fast alle $i$ :

$$
\begin{aligned}
& c \geqq\left|\sum f_{j}\left(x_{j}\right)-b\right|=\left|\sum g_{j i}\left(T_{i} x_{j}\right)-b\right|=\left|\sum g_{j}\left(x_{j}\right)-b\right|, \\
& c \geqq \lim _{U}\left|g_{j i}\left(x_{j i}\right)-b\right|, \quad \text { wenn }\left(x_{j i}\right)_{u}=x_{j} \text { ist }-
\end{aligned}
$$

(vgl. die Definition von $p(\varepsilon, i)$ ). Da wenigstens eins der Elemente $x_{j}$ von Null verschicden ist, gilt (6) für fast alle $i$.

2. Fall: Es sei $\dot{p}=\left(\dot{d}\left(\sum a_{j} g_{j}+f, Z^{\prime}\right) \leqq c\right)$.

1. Schritt: Für fast alle $i$ und für beliebiges $\delta>0$ haben die Mengen

$$
\text { - } D_{i}=K_{i}^{+}+\left\{g \in E_{i}^{*}:\|g\|^{\prime} \leqq c+\delta\right\}
$$

und

$$
\dot{H}_{i}=\left\{\dot{g} \in E_{i}^{*}: g\left(\dot{T}_{i} x\right)=\sum a_{j} f_{j}(x)+f_{i}\left(T_{i} x\right), \quad\|g\| \leqq\left\|\sum a_{j} f_{j}+f\right\|+1\right\}
$$

nichtleeren Durchschnitt (wobei $\left(f_{i}\right)_{U}=f$ gilt, vgl. die Definition v'on $p(\varepsilon, i)$ ). Angenommien dies sei falsch. Dann können diese Mengen durch eine $w^{*}$-abgeschlossene Hyperebene getrennt werden, d. h. für fast alle $i$ existieren Elemente $y_{i} \in E_{i}$ mit $\left\|y_{i}\right\|=1$, so daß gilt:

$$
\begin{aligned}
& \sup \left\{\operatorname{Re} g\left(y_{i}\right): g \in D_{i}\right\}<\inf \left\{\operatorname{Re} g\left(y_{i}\right): g \in H_{i}\right\}, \\
& y_{i} \in-K_{i+}^{+}=-\bar{K}_{i}, \sup \left\{\operatorname{Re} g\left(y_{i}\right): g \in D_{i}\right\}=c+\delta .
\end{aligned}
$$

Also kann man auch $z_{i} \in-K_{i}$ nit $\left\|z_{i}\right\|=1$ so wählen, da.ß

$$
\inf \left\{\operatorname{Re} g\left(z_{i}\right): g \in H_{i}\right\}>c+\delta
$$


gilt. Sei $T_{i}^{\prime}$ eine Fortsetzung von $T_{i}$ auf die lineare Hülle von $M$ und $\left(z_{i}\right)_{U}=z$ mit $\left(T_{i}^{\prime} z\right)_{U}=z$ (Lemma 6$)$, und sei $g_{i}$ eine Hahn-Banach-Fortsetzung von

$$
T_{i}^{\prime}\left(\operatorname{lin}(M \cup\{z\}) \in x \rightarrow-\sum a_{j} f_{j}\left(T_{i}^{\prime-1} x\right)+\dot{f}_{i}(x)\right. \text {. }
$$

Dann folgt im Widerspruch zur Voraussetzung für fast alle $i$

$$
\begin{aligned}
& g_{i} \in H_{i},\left(g_{i}\right)_{U}(z)=\sum a_{j} f_{j}(z)+f(z) \geqq c+\delta, \\
& d\left(\Sigma a_{j} f_{j}+f, Z^{\prime}\right) \geqq c+\delta .
\end{aligned}
$$

2. Schritt: Wir wählen ein festes $k$, so daß $a_{k} \neq 0$ ist. Nach dem Resultat des 1. Schrittes ist es möglich, Funktionale $g_{i} \in E_{i}{ }^{*}$ so festzulegen, daß für fast alle $i$ gilt $g_{i} \in D_{i} \cap H_{i}$. Falls $j \neq k$ ist, so bestimmen wir $g_{j i}$ als Hahn-Banach-Fortsetzúng . von $h_{j i}$. Danach setzen wir $g_{k i}=a_{k}^{-1}\left(g_{i}-f_{i}-\sum_{j \neq k} g_{j i}\right)$. Dann folgt $d\left(\sum a_{j} g_{j i}+f_{i}, K_{i}^{+}\right)$ $\leqq c+\delta$ für fast alle $i$. Für genügend kleines $\delta$ folgt daraus (6).

3. Fall: Es sei $p=\left(g_{j} \in Z^{\prime}\right)$.

Die Behauptung folgt aus dem zweiten Fall für die Formel

$$
d\left(g_{j}, Z^{\prime}\right) \leqq 0 .
$$

4. Fall: Es sei $p \doteq\left(\left\|\sum a_{j} g_{j}+f\right\| \leqq c\right)$.

Ersetzt man' $K_{i}$ durch $E_{i}^{\prime}$, so erhält man $Z=Z^{\prime}=\{0\}$ und diè Behauptung folgt durch Anwendung des zweiten Falles

Lemma 8: Seien $\delta>0$, eine endliche Formelmenge $Q \subset P$ und eine Folge $\left(f_{j}\right)$ so vorgegeben; duß $\left(f_{j}\right) \models Q$ ist. Dann existiert für fast jedes $i \in I$ eine Folge $\left(g_{j}\right)$ mit $\left(g_{j}\right) \models Q(\delta, i)$ :

Beweis: Da in $Q$ nur endlich viele Elemente. $g_{j}$ wirklich auftreten, kann man $m<\infty$ annehnien. Angenommen die Behauptung sei falsch. Dann haben für fast alle $i$ dic Mengen

$$
\begin{aligned}
& C_{p i} \doteq\left\{\left(g_{1}, \ldots, g^{m}\right) \in\left(E_{i}^{*}\right)_{m}:\left(g_{j}\right) \models\{p(\delta, i)\}\right\} \quad\left(p \in Q \backslash P_{4}\right) ; \\
& C_{0 i}=\left\{\left(g_{1}, \ldots, g^{m}\right) \in\left(E_{i}{ }^{*}\right)_{m}: \sum\left\|g_{j}\right\|^{2} \leqq \sum\left\|f_{j}\right\|^{2}+1\right\}, \\
& C_{1 i}=\left\{\left(g_{1}, \ldots, g^{m}\right) \in\left(E_{i}^{*}\right)_{m}:\left(g_{j}\right) \models\{p(\delta, i)\} \quad \text { falls } p \in Q \cap P_{4} \text { ist }\right\}
\end{aligned}
$$

leeren Durchschnitt. Nach Lemma $4 \mathrm{~b})$ können trennende Elemente $x_{s i} \in\left(E_{i}\right)^{m}$ und Zahlen $b_{s i}$ bestimmt werden. Zusätzlich kann man noch voraussetzen, da 3 $\sum_{\dot{s}}\left(\left\|x_{s i}\right\|+\left|b_{s i}\right|\right)=1$ ist. Dann werden aber die Mengen $J\left(C_{p_{i}}\right)_{U}, J\left(C_{0 i}\right)_{U}^{-}$und $J\left(C_{1 i}\right)_{U}$ und folglich auch ihre $\mathrm{w}^{*}$-Abschlüsse durch $x_{s}=\left(x_{s i}\right)_{U}$ und $b_{s}=\lim _{U} b_{s i}$ getrennt. Im Widerspruch dazu folgt aber aus Lemma $7, \mathrm{da} B\left(f_{1}, \ldots, f_{m}\right)$ innerer Punkt der w*-Abschliisse von $\left(C_{p i}^{\prime}\right)_{U}\left(p \in Q \backslash P_{4}\right)$ und von $\left(C_{0 i}\right)_{U}$ ist und,auch im w*-Abschlu $B$ von $\left(C_{1 i}\right)_{U}$ liegt

\section{Beweis der Theoreme 3 und 4}

Beweis von Theorem 3: Sei $\left(f_{j}\right)_{j-1}^{m}$ eine linear unabhängige Folge in $L$, déren lineare Hiille eine in $L \cap F$ dichte 'Teilmenge von $L \cap F$ enthält und selbst in $L$ dicht liegt. Außerdèm sei $M_{n}$ eine aufsteigende Folge endlichdimensionaler Teilräume von $M$, deren Vereinigung dicht in $M$ ist. Durch wiederholte Anwendung von Lemma 1 wird eine aufsteigende Folge endlicher Formelmengen $Q_{n} \subset P$ und eine monoton 
fallende Nullfolge $\left(\delta_{n}\right)$ so bestimmt, $\mathrm{da} B\left(f_{j}\right) \models Q_{n}$ ist und da $B$ für den auf der linearen Hülle $L_{n}$ von $\left\{f_{1}, \ldots, f_{n}\right\}$ durch $T_{n} f_{j}=g_{j}$ definierten linearen Operator folgendes gilt: Aus $\left(g_{j}\right) \models Q_{n}\left(\delta_{n}\right)$ und aus $f \in L_{n}, x \in M_{n}$ folgen (1), (2) und (3), wobei $0=1 / n$ ist. Zusätzlich kann noch erreicht werden, daß für eine abzählbare in $L \cap F$ dichte Teilmenge von Elementen der Form

$\circ=\sum_{\text {endl. }} \lambda_{j} f_{j}$,

die selbst in $F$ liegen, jeweils die Formel

$$
\left(\left\|\sum \lambda_{j} g_{j}-f\right\| \leqq 0\right)
$$

zu $\cup Q_{n}$ gehört. Für jedes $i$ sei

$$
n(i)=\sup \left\{n+1: \exists\left(g_{j}\right) \text { mit }\left(g_{j}\right) \risingdotseq Q_{n}(1 / n, i)\right\} .
$$

Weiterhin sei für $n<n(i)$ eine Folge $\left(g_{j i}^{n}\right)_{j-1}^{m}$ mit

$$
\left(g_{j i}^{n}\right) \models Q_{n}(1 / n, i)
$$

festgelegt. Definiert man mit Hilfe eines freien O̊ltrafilters $V$ auf der Menge der natürlichen Zahlen

$$
g_{j i}= \begin{cases}g_{j i 1}^{n(i)-1} ; & \text { wenn } n(i)<\infty \text { ist } \\ \mathrm{w}^{*}-\lim _{n . V} g_{j i}^{n} ; & \text { wenn } n(i)=\infty \text { ist }\end{cases}
$$

so folgt $\left(g_{j i}\right) \models D_{n}(1 / n, i)$, wenn nur $n<n^{\prime}(i)$ ist. Da bei beliebigem festem $n$ aus Lemma 8 für fast alle $i$ folgt $n(i)>n$, erhält man für $\left(g_{j}\right)=\left(g_{j i}\right)_{U}$ und für beliebiges $n$

$$
\left(g_{j}\right) \models Q_{n}(1 / n) \text {. }
$$

Wird nun $T$ auf der linearen Hülle von $\left\{f_{j}\right\}_{j-1}^{m}$ durch $T f_{j}=J^{-1} g_{j}$ definiert, so ist $T$ isometrisch. Sei $S$ der AbschluB von $T$. Dann sind die Behauptungen 1, 2 und 3 des Theorems erfüllt. Behauptung 4 ist auch erfüllt, da $J S f=f$ für eine dichte Teilmenge von $L \cap J\left(E_{i}{ }^{*}\right)_{U}$ aus $\left(g_{j}\right) \models \cup Q_{n}(0)$ folgt. Es muß nur noch $J\left(K_{i}{ }^{+}\right)_{U}=\left(K_{i}\right)_{U}{ }^{+} \cap F$ gezeigt werden. Sei $f=\left(f_{i}\right)_{U} \in\left(K_{i}\right)_{U}{ }^{+}$und sei $Q$ die Menge der beiden Formeln $\left(\left\|g_{1}-f\right\| \leqq 0\right),\left(g_{1} \in Z^{\prime}\right)$, wobei $m=1$ ist. Da $f$ diese Formeln erfüllt, gehört nach Lemma 8 für jedes feste $n$ die Menge der $i$, für die ein $h_{i}{ }^{n}$ mit $\left(h_{i}{ }^{n}\right) \models Q(1 / n, i)$ existiert, zum Ultrafilter. Wie oben findet man ein $h$ mit $(h) \models Q(0)$. Das bedeutet aber $h=f$ und $h \in Z=\dot{J}\left(K_{i}^{+}\right)_{U}$

Beweis von Theorem 4: Wir wählen $f_{1}, \ldots, f_{m}$ und $e_{1}, \ldots, e_{r}$ wie im Beweis v̀n Theorem 2, d. h. die $f_{j}$ bilden eine Basis von $L$ aus Atomen, $e_{1}, \ldots, e_{r}, f_{n+1}, \ldots, f_{m}$ bilden eine Basis aus Atomen für $L \cap F$ und es gibt Zahlen $\lambda_{s j} \in\{0,1\}$, so daß\} $e_{s}=\sum_{j=1}^{n} \lambda_{s j} f_{j}$ gilt. Sei $\left(M_{l}\right)$ eine aufsteigende Folge endlichdimensionaler Unterräume von $M$, deren Vereinigung dicht in $M$ ist. Mit Hilfe von Lemma 1 läßt sich eine Folge endlicher Teilmengen $Q_{l} \subset P_{1}$ und eine monotone Nullfolge positiver Zahlen $\theta_{l}$ so bestimmen, $\operatorname{da} \beta\left(f_{j}\right) \models Q_{l}$ und $Q_{l} \subset Q_{l+1}$ ist, und daß aus $\left(g_{j}\right) \models Q_{l}\left(\theta_{l}\right)$ für den auf $L$ durch $T f_{j}=g_{j}$ definierten linearen Operatơr fọtgt

$$
\begin{array}{ll}
(1-1 / l)\|f\| \leqq\|T f\| \leqq(1+1 / l)\|f\| & (f \in L), \\
|(T f-f) .(x)| \leqq 1 / l\|f\|\|x\| &
\end{array}
$$

Auf Grund von Lemma 3 lassen sich nun endliche Teilmengen $R_{l} \subset P$ und eine monotone Nullfolge positiver Zahlen $\delta_{l}$ so finden, daß $Q_{l} \subset R_{l} \subset R_{l+1}$ und $\left(f_{j}\right) \models R_{l}$ 
ist, und daß aus $\left(h_{j}\right)=R_{l}^{\prime}\left(\delta_{l}\right)$ und aus (4) mit $\delta=\delta_{l}$ folgt $\left(g_{j}\right) \models Q_{l}\left(\theta_{l}\right)$. Außerdem kann noch erreicht werden, daß $P_{4} \subset R_{1}$ gilt und daß auch folgende Formeln żu $R_{1}$ gehören :

$$
\begin{array}{ll}
\left\|g_{j}\right\| \leqq\left\|f_{j}\right\| & (1 \leqq j \leqq m), \\
\left\|\dot{g}_{j}-f_{j}\right\| \leqq 0, & (n<j \leqq m), \\
\left\|\sum_{j=1}^{n} \lambda_{s j} g_{j}-\dot{e}_{s}\right\| \leqq 0 & (1 \leqq s \leqq r) .
\end{array}
$$

Für $i \in I$ sei

$$
l(i)=\sup \left\{l+1: \exists\left(h_{j}\right) \cdot \operatorname{mit} \quad\left(h_{j}\right) \models R_{l}\left(\delta_{l}, i\right)\right\} .
$$

Für $l<l(i)$ sei $\left(h_{j i}^{l}\right)_{j-1}^{m}$ so gewählt, $\mathrm{da} B\left(h_{j i}^{l}\right) \models{ }^{\prime} R_{l}\left(\delta_{l}, i\right)$ ist. Weiterhin'sei

$$
h_{j i}= \begin{cases}h_{j i}^{l(i)-1}, & \text { wenn } l(i)<\infty \text { ist } \\ w^{*}-\lim _{l, V} h_{j i}^{l}, & \text { wenn } l(i)=\infty \text { ist. }\end{cases}
$$

Ist $l<l(i)$, so gilt $\left(h_{j i}\right) \models R_{l}\left(\delta_{l}, i\right)$. Aus Lemma 8 folgt bei festem $l$, da $B$ für fast alle $i$ gilt $l(i)>l$. Deshalb existiert $h_{j}=\left(h_{i i}\right)_{U}$ und es gilt $\left(h_{j}\right) \vDash \bigcup_{l} R_{l}\left(\delta_{l}\right)$ und folglich auch $\left(h_{j}\right) \models \cup R_{l}(0)$. Für $l<l(i)$ werden Elemente $g_{j i}^{l} \in E_{i}^{*}$ definiert. Dabei werden in Abhängigkeit von $j$ drei Fälle unterschieden.

1. Fall: Es sei $n<j \leqq m$. Wir setzen

$$
g_{j i}^{l}=\left(h_{j i}-\delta_{l}^{1 / 2} \sum_{k \neq j} h_{k i}\right)^{+} \text {. }
$$

2. Fall: Es existiert ein $s$ mit $f_{j} \leqq e_{s}\left(\right.$ d. h. $\left.\lambda_{s i}=1\right)$. Wir setzen

$$
g_{j i}^{l}=\sup _{q \in \mathbf{N}}\left(q\left(h_{j i}-\delta_{l}^{-1 / 2} \sum_{k \neq j} h_{k i}\right)^{+}\right) \wedge \sum_{k=1}^{n} \lambda_{s k} h_{k i} .
$$

3. Fall: Es sei $1 \leqq j \leqq n$ und $f_{j} \wedge \sum e_{s}=0$. Wir setżen

$$
\left.g_{j i}^{l}=\left(h_{j i}-\delta_{l}^{-1 / 2} \sum_{k \neq j} h_{k i}\right)\right)^{+} .
$$

Beriucksichtigt man, daB Disjunktheit bei monotonen Grenzwerten erhalten bleibt, so kann man wie beim Beweis von Theoren 2 zcigen, da $\beta g_{j i}^{l} \wedge g_{k i}^{l}=0$ ist falls $1 \leqq j \leqq n, 1 \leqq k \leqq m ; j \neq k$ gilt.'Definiert man $\left(g_{j i}^{l}\right)_{U}=g_{j}{ }^{l}$, so folgt auf Grund der Bemerkung nach Lemma 3 , daß die Elemente $\left(g_{j}^{l}\right)_{j-1}^{m}$ die Ungleichungen (4) mit $\delta=\delta_{l}$ erfüllen. Es folgt also $\left(g_{j}{ }^{l}\right) \models Q_{l}\left(\theta_{l}\right)$. Da. jede. Formel $p \in \cdot Q_{l}$ die Gestalt $\left\|\sum a_{j} g_{j}\right\| \leqq c$ bzw. $\left|\sum g_{j}\left(\left(x_{j i}\right)_{v}\right)-b\right| \leqq c$ hat, folgt $\cdot\left(g_{j i}^{l}\right) \wedge=Q_{l}\left(20_{l}, i\right)$ für fast alle $i$, denn es gilt

$$
\sum g_{j}^{l}\left(\left(x_{j i}\right)_{U}\right)=\lim _{U} \sum g_{j i}^{l}\left(x_{j i}\right) \quad \text { und } \quad\left\|\sum a_{j} g_{j}{ }^{l}\right\|=\lim _{U}\left\|\sum a_{j} g_{j i}^{l}\right\|
$$

Für jedes feste $i$ sei $V_{i}$ ein (möglicherweise auch trivialer) Ültrafilter auf dèr Menge

$$
\left\{q \in \mathbf{N}:\left(g_{j i}^{q}\right) \models Q\left(20_{q}, i\right)\right\},
$$

der gegen das Supremum $q(i)$ dieser Menge strebt. Es sei gleich bemerkt, daß bei festem $q$ für fast alle $i$ gilt $g(i)>q$. Insbesondere ist die Definition von $V_{i}$ für fast 
alle $i$ möglich. Sei

$$
g_{j i}=\mathrm{w}^{*} \lim _{q, v_{i}} g_{j i}^{q}, \quad g_{j}=\left(g_{j i}\right)_{U} .
$$

Es gilt dann $\left(g_{j}\right) \models \cup Q_{q}\left(2 \theta_{q}\right)$ und folglich auch $\left(g_{j}\right) \models \cup Q_{q}(0)$. Der Operator $S$ sei durch die Formeln $S f_{j}^{q}=J^{-1} g_{j}$ definiert. Es folgen dann sofort die Behauptungen 1 'und 2 des Theorems.

Für $n<j \leqq m$ folgt aus $\left(h_{j i}\right) \models R_{l}\left(\delta_{l}, i\right)$

$$
\left\|h_{j i}-f_{j i}\right\| \leqq \delta_{l}, \ldots\left\|g_{j i}^{l}-h_{j i}\right\| \leqq \delta_{l}^{1 / 2} \Sigma\left\|h_{j i}\right\| .
$$

Deshalb gilt für diese $j$ auch $g_{j}=f_{j}$. Ist $1 \leqq j \leqq n, 1 \leqq k \leqq m$ und $j \neq k$, so gilt. $g_{j i}^{l} \wedge g_{k i}^{l}=0$. Es folgt, da $\beta$ die Elemente $g_{j}$ positiv und paarweise disjunkt sind und daß also Behauptung 3 gilt.

Da für $f_{j} \leqq e_{s}$ und für $l<l(i)$ nach Definition $g_{j i}^{l}$ die Projektion von $\sum_{k=1}^{n} \lambda_{s k} h_{k i}$ auf

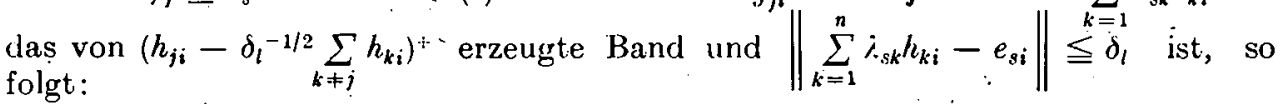

$$
\begin{aligned}
& \left(\sum_{k=1}^{n} \lambda_{s k} g_{j i}^{l}\right) \wedge\left(\sum_{k=1}^{n} \lambda_{s k}\left(h_{j i}-g_{j i}^{l}\right)\right)=0 \\
& \left(\sum_{k=1}^{n} \lambda_{s k} g_{j i}\right) \wedge\left(\sum_{k=1}^{n} \lambda_{s k i}\left(h_{j i}-g_{j i}\right)\right)=0 \\
& \left(S e_{s}\right) \wedge\left(e_{s}-S e_{s}\right)=0 .
\end{aligned}
$$

Daraus folgt Behauptung 4 des Theorems

\section{Beispiele}

In [1] sind drei Beispiele angegeben, die zeigen, da $B$ in den Behauptungen 2 und 5 von Theorem 2 die positive Zahl $\varepsilon$ nicht durch Null ersetzt werden kann und daß in allgemeinen Banachverbänden Behauptung 5 nicht erfüllbar ist. Wir werden jetzt einige weitere abgrenzende Beispiele bringen.

Beispiel 1: Fiir die endliche Darstellbarkeit eines Dualraumes $\dot{E}^{*}$ in seinem Unterraum $F$ ist die w*-Dichtheit der Einheitskugel von $F$ in der Einheitskugel von $E^{*}$ nicht hinreichend. Bezeichnet man mit $y_{l}=\left(y_{l n}\right)_{n-1}^{\infty}$ die Folge der Rademacherfolgen, d. h. setzt man $y_{l n}=$ sign $\sin \left(2^{-l} \pi(2 n+1)\right)$, so kann man Elemente $z_{l}$ von $l_{\infty}$ so finden, daß sich die Folgen $z_{l}$ und $y_{l}$ jeweils nur in endlich vielen Gliedern unterscheiden und da $\beta\left\{\dot{z}_{l}\right\}$ eine $w^{*}$-dichte Teilmenge der Einheitskugel von $l_{\infty}=l_{1}{ }^{*}$ ist. Auf Grund der Figenschaften der Rademacherfolgen gilt für rẹelle Zahlen $\lambda_{l}$

$$
\left\|\sum \dot{i}_{l} z_{l}\right\| \geqq\left\|\Sigma \dot{\lambda}_{l} \dot{y}_{l}\right\|=\sum\left|\dot{i}_{l}\right| \text {. }
$$

Deshalb ist die abgeschlossene lineare Hülle $F$ von $\left\{z_{l}\right\}$ (auch im Falle komplexer Banachräume) isomorph zu $l_{1}$. Ist nun ein Unterraum $H$ von $F$-isomorph zu $l_{\infty}^{n}$ (d. h. existiert ein Operator $T: l_{\infty}^{n} \rightarrow H$ mit $\left\|T^{\prime}\right\|\left\|T^{-1}\right\| \leqq c$ ), so ist die identische Abbildung von $l_{\infty}^{n}$ in folgender Weise faktorisierbar:

$$
\text { - } l_{\infty}^{n} \stackrel{T}{\rightarrow} H \hookrightarrow F \stackrel{\dot{s}}{\rightarrow} l_{\infty}^{n}, \quad\left\|T^{\prime}\right\|\|S\| \leqq c .
$$

Dann ist aber $S^{*} T^{*}$ eine Faktorisierung der identischen Abbildung von $l_{1}{ }^{n}$ durch den $P_{\lambda}$-Raun $F^{*}$. Daraus, daß die Projektionskonstanten von $l_{1}{ }^{2}$ gegen unendlich streben (vgl. [5]), folgt, da $\beta l_{\infty}$ nicht in $F$ endlich darstellbar ist. 
Beispiel 2: Unter den Voraussetzungen yon Theorem 1 kann selbst bei abgeschlossenem $K$ nicht erreicht werden, daß $S\left(L \cap K^{++}\right) \subset K$ gilt, wenn auBerdem Ker $S=\{0\}$ verlangt wird. Sei

$$
x_{l n}= \begin{cases}1, & \text { wenn } l \leqq n \leqq l+1 \text { ist } \\ 0, & \text { wenn } n<l \text { oder } n>l+1 \text { ist. }\end{cases}
$$

Es werden die Elemente $x_{l}=\left(x_{l n}\right)_{n-1}^{\infty}$ in $l_{1}=\left(c_{0}\right)^{*}$ betrachtet. Sei $K=\left(\left\{x_{l}\right\}_{l-1}^{\infty}\right)_{+}$. $K$ enthält keine Gerade, weil die $w^{*}$-abgeschlossene lineare Hülle von $\left\{x_{l}\right\}$ mit $l_{1}$ zusammenfällt. Andererseits liegt die lineare Hülle $L$ von $\left((-1)^{n}\right)_{n=1}^{\infty}$ in $\cdot K^{++}$, denn die Folgen $\left(f_{l n}\right)_{n-1}^{\infty}$ mit

$$
f_{i n} \begin{cases}=(-1)^{l-n}, & \text { wenn } n \leqq l \text { ist } \\ =0, & \text { wenn } n>l \text { ist }\end{cases}
$$

liegen in $K$. Aus $S L \subset K$ wiirde nun folgen $S(L)=\{0\}$.

Beispiel 3: Unter den Voraussetzungen von 'l'heorem 1 kann nicht erreicht werden, daß $\|S f\|=\|f\|$ gilt. Der reelle Raum $c_{0}{ }^{* *}\left(=l_{\infty}\right)$ enthält einen zweidimensionalen - Euklidischen Raum, z. B. die lineare Hülle von $\left(\cos t_{n}\right)_{n=1}^{\infty}$ und $\left(\sin t_{n}\right)_{n-1}^{\infty}$, wobei $\left(t_{n}\right)$ eine in $[0,2 \pi]$ dichte Folge reeller Zahlen ist. Der Raum $c_{0}$ selbst enthält jedoch als polyedrischer Raum (vgl. [8]) keinen zweidimensionalen Euklidischen Unterraum.

Bemerkung: Unter den Voraussetzungen von Theorem 2 kann aụch nicht $\|S f\|=\|f\|$ erreicht werden, weil es polyedrische Banachverbände gibt, so da $\beta$ der zweidimensionale Euklidische Raum Unterverband des Bidualen ist.

Beispiel 4: Unter den Voraussetzungen von Theorem 4 ist $J\left(E_{i}{ }^{*}\right)_{U}$ im allgemeinen nicht $\sigma$-ordnungsvollständig und es ist möglich, dả die Summe 'zweier positiver disjunkter Elemente von $\left(E_{i}\right)_{U}{ }^{*} z, 11 J\left(E_{i}{ }^{*}\right)_{U}$ gehört, während jedes Einzelne dieser Elemente nicht in $J\left(E_{i}{ }^{*}\right)_{i}$ liegt. Als Indexmenge $I$ sei die Menge der natiirlichen Zahlen mit cinem freien Ultrafilter $U$ gewählt. Sei $E_{i}=l_{1}$ und seien $e_{n}$ die kanonischen Bilder der üblichen Koordinatenbasis von $c_{0}$ in $l_{1}^{*}=c_{0}^{* *}$. Es werden folgende Elemente betrachtet:

$$
\begin{aligned}
f_{k i} & =\sum_{n=1}^{k} e_{n} \in E_{i}{ }^{*}, \quad g_{i}=\sum_{n=1}^{i} e_{n} \in E_{i}, \quad e=w^{*} \lim _{k \rightarrow \infty} \sum_{n=1}^{k} e_{n}, \\
-\quad f_{k} & =\left(f_{k i}\right)_{U} \in\left(E_{i}\right)_{U}{ }^{*}, g=\left(g_{i}\right)_{U} \in\left(E_{i}\right)_{U}{ }^{*}, \quad h=\sup _{k} f_{k} \in\left(E_{i}\right)_{U}{ }^{*} .
\end{aligned}
$$

Bei festem $k$ gilt $f_{k i} \wedge\left(g_{i}-f_{k i}\right)=0$ für fast alle $i$. Es folgt

$$
f_{k} \wedge\left(g-f_{k}\right)=0, \quad h \wedge(g-h)=0 .
$$

Deshalb reicht es zu beweisen, daß $h \notin J\left(E_{i}^{*}\right)_{U}$ ist. Angenommen, es ist $h=\left(h_{i}\right)_{i}$. Dann gilt $\left(h_{i} \wedge f_{k i}\right)_{U}=h \wedge f_{k}=f_{k}$. Bei festem $k$ folgt $h_{i} \geqq f_{k i}-k^{-1} e$ für fast alle $i$. Deshalb kann man eine Folge $\left(I_{k}\right)$ von Elementen des Ultrafilters so finden, da $\beta$ fiir $i \in I_{k}$ gilt $h_{i} \geqq f_{k i}-k^{-1} e$. Zusätzlich kann $\cdot I_{k+1} \subset I_{k}$ und $k \notin I_{k}$ erreicht werden. Setzt man $d_{i}=\left(1-k^{-1}\right) e_{k} \in E_{i}^{*}$ für $i \in I_{k} \backslash I_{k+1}$, so folgt

$$
f_{k} \wedge\left(d_{i}\right)_{U}=0, \quad\left(d_{i}\right)_{U} \leqq h, \quad\left\|\left(d_{i}\right)_{U}\right\|=1,
$$

im Widerspruch zur Beweisannahme.

Bemerkung: Es ist unbekannt, ob in Theorem 4 zusätzlich erreicht werden kann, daß für $f \in J F \cap L$ gilt $J S f=f$. 


\section{IITERATUR}

[1] Bernad, S. J.: A unified approach to the principle of local reflexivity. In: Notes in Banach spaces. Univ. of Texas: Austin 1981.

[2] Conroy, J. L., and L. C.. Moore Jr.: Local reflexivity in Banach lattices. Preprint: Duke University: Durham.

[3] Dean, D. W.: The equation $L\left(E, X^{* *}\right)=L(E, X)^{* *}$ and the principle of local reflexivity. Proc. Amer. Math. Soc. 40 (1973), 146-148.

[4] ГейлеР, В.А., и И. И. ЧучАев: Общий принцип локальной рефлексивности и его. применения в теории двонственности конусов. Сиб. мат. журиал 23,1 (1982), 32-43.

[5] Grünbaun, B.: Projection constants. Trans. Amer. Math. Soc. 95 (1960), 451-465.

[6] Heinrich, S.: Ultraproducts in Banach space theory. J. Reine Angew. Math. 313 (1980), $72-104$.

[7] Johnson, W. B., Rosenthal, H. P., and M. Zipin: On bases, finite dimensional decompositions and weaker structures in Banach spaces. Israci J. Math. 9 (1971), 488-506.

[8] KLEe, V.: Polyhedral sections of convex bodies. Acta Math. 103 (1960), 243-267.

[9] Kürstex, K.-D. : Local Duality of Ultraproducts of Banach Lattices. In: Banach Space Theory and its Applications. Proceedings, Bucharest 1981, 137-142, Springer-Verlag: Berlin-Heidelberg-New York-Tokyo 1983.

[10] Кюрстен, К.-Д.: О пекоторых попросах А. ПичА II. Теория функций, функц. анализ и их прил. $\mathbf{2 9}(\mathbf{1 9 7 8 )}, 61-73$.

[11] Lindexstrauss, J., and H. P. Rosenthal: The $\mathfrak{Q}_{p}$ spaces. Israel J. Math. 7 (1969), $325-349$.

[12] Scha EFER, H. H.: Topological vectór spaces. Macmillan: New York 1966.

[13] Schaefer, H. H.: Banach lattices and positive operators. Springer-Verlag: BerlinHeidelberg-New York 1974.

[14] Strkx, J.: Ultraproducts and local properties of Banach spaces. Trans. Amer. Math. Soc. 240 (1978), $231-252$.

[15] ZeIdLER, E.: Vorlesungen über nichtlineare Funktionalanalysis III - Variationsmethoden und Optimierung -. BSB B. G. Teubner Verlagsgesellschaft: Leipzig 1977.

Manuskripteingang: 10.12.1982

VERFASSER :

Dr. KLAUS-DetLef Kürsten ,

Sektion Mathematik der Karl-Marx-Universität

DDR-7010 Leipzig, Karl-Marx-Platz 